\title{
La diététique et la physiothérapie face au problème des frontières interprofessionnelles (1950-1980)
}

\section{Aline Charles et Nadia Fahmy-Eid}

Volume 47, numéro 3, hiver 1994

URI : https://id.erudit.org/iderudit/305246ar

DOI : https://doi.org/10.7202/305246ar

Aller au sommaire du numéro

Éditeur(s)

Institut d'histoire de l'Amérique française

ISSN

0035-2357 (imprimé)

1492-1383 (numérique)

Découvrir la revue

Citer cet article

Charles, A. \& Fahmy-Eid, N. (1994). La diététique et la physiothérapie face au problème des frontières interprofessionnelles (1950-1980). Revue d'histoire de l'Amérique française, 47(3), 377-408. https://doi.org/10.7202/305246ar
Résumé de l'article

La diététique et la physiothérapie font partie de ces nouvelles professions paramédicales qui se multiplient à partir des années 1950 et qui présentent un profil différent de celui des professions libérales traditionnelles puisqu'elles sont salariées, très majoritairement occupées par des femmes et ont un cadre de pratique institutionnel. Parce que l'État leur a accordé un droit au titre réservé, mais non au monopole de pratique, la diététique et la physiothérapie se verront plus démunies face aux problèmes des frontières interprofessionnelles qui surgissent entre elles et la profession médicale, d'une part, et entre elles et les professions connexes du paramédical, d'autre part. Cet article analyse les conditions d'émergence et les modalités des stratégies de défense mises au point par les deux professions concernées.
Tous droits réservés @ Institut d'histoire de l'Amérique française, 1994

Ce document est protégé par la loi sur le droit d'auteur. L'utilisation des services d'Érudit (y compris la reproduction) est assujettie à sa politique d'utilisation que vous pouvez consulter en ligne.

https://apropos.erudit.org/fr/usagers/politique-dutilisation/ 


\title{
LA DIÉTÉTIQUE ET LA PHYSIOTHÉRAPIE FACE AU PROBLÈME DES FRONTIÈRES INTERPROFESSIONNELLES (1950-1980)
}

\author{
ALINE CHARLES \\ NADIA FAHMY-EID \\ Département d'histoire \\ Université du Québec à Montréal
}

\section{RÉSUMÉ}

La diététique et la physiothérapie font partie de ces nouvelles professions paramédicales qui se multiplient à partir des années 1950 et qui présentent un profil différent de celui des professions libérales traditionnelles puisqu'elles sont salariées, très majoritairement occupées par des femmes et ont un cadre de pratique institutionnel. Parce que l'État leur a accordé un droit au titre réservé, mais non au monopole de pratique, la diététique et la physiothérapie se verront plus démunies face aux problèmes des frontières interprofessionnelles qui surgissent entre elles et la profession médicale, d'une part, et entre elles et les professions connexes du paramédical, d'autre part. Cet article analyse les conditions d'émergence et les modalités des stratégies de défense mises au point par les deux professions concernées.

\section{ABSTRACT}

Dietetics and physiotherapy are part of the new paramedical professions that have multiplied since the middle of the $20^{\text {th }}$ century. Salaried, practicing within an institutional framework and based on a heavily female membership, they present a quite different profile from the one which characterized the traditional liberal professions. Because the State allowed dietetic and physiotherapy a reserved title but not a monopoly of practice, they have faced many critical problems of interprofessional boundaries with the medical profession as well as with other paramedical occupations. This article will focus on some of the defensive strategies developed by these two professions in such a context.

À partir de la deuxième moitié du $\mathrm{XX}^{\mathrm{e}}$ siècle, diététistes et physiothérapeutes affrontent au Québec un contexte de bouleversements majeurs. Le secteur de la santé connaît une expansion sans précédent pour répondre à une demande croissante de services. L'augmentation de cette demande, alliée au développement des connaissances 
biomédicales, multiplie le nombre de groupes professionnels qui se côtoient dans le secteur de la santé. Tant les spécialités médicales que les professions paramédicales se font plus nombreuses. Au début des années 1970 cependant, l'ampleur du fardeau financier que représente l'appareil sociosanitaire pousse l'État québécois à créer un nouveau groupe socioprofessionnel: celui des techniciennes et des techniciens, beaucoup moins coûteux à former et à employer que des professionnels; l'apparition des techniques doit aussi être située dans le contexte de la réforme du secteur de l'éducation et de la création des cégeps. Ces techniques surgissent la plupart du temps sur un terrain déjà occupé par des professions plus anciennes et au statut mieux reconnu. Les diététistes et les physiothérapeutes voient ainsi survenir en 1969 des «techniciennes en diététique» et des «techniciennes en réadaptation physique», ce qui annonce le morcellement de leur champ de pratique $^{1}$.

Depuis les années 1950, le secteur de la santé s'est donc transformé en une mosaïque de plus en plus complexe d'occupations. En 1980 , les médecins peuvent choisir parmi plus de 30 spécialités différentes, tandis qu'une vingtaine de professions et près d'une quinzaine de techniques se partagent le champ du paramédical ${ }^{2}$. Or, la logique professionnelle veut que chaque groupe tente d'occuper un «territoire» sur lequel il puisse faire reconnaître des droits de quasipropriété. Dans un contexte où les occupations en présence se multiplient, la concurrence et les conflits interprofessionnels auront tendance à s'aggraver.

Nous montrerons comment les diététistes et les physiothérapeutes n'échapperont pas à la dynamique de la concurrence professionnelle. Elles se situent, en effet, à la fois dans un rapport de verticalité, entre médecins et techniciennes, et dans un rapport d'horizontalité, avec des professions connexes telles que celle des infirmières. Nous verrons comment nos deux professions se négocient un «territoire» propre dans cet univers complexe des rapports interprofessionnels.

1. Les infirmières ont été confrontées au même problème avec l'apparition des infirmières auxiliaires. Le morcellement n'est cependant pas spécifique au secteur de la santé ni aux professions féminines: ingénieurs, avocats et dentistes, par exemple, voient survenir dans leur champ des «techniciens en génie civil», des «auxiliaires de la justice» et des «techniciens dentaires». Seuls les médecins semblent échapper à ce phénomène.

2. G. Dussault, «Les effectifs sanitaires au Québec», J. Dufresne et al., dir., Traité d'anthropologie médicale, l'institution de la santé et de la maladie (Presses de l'Université du Québec, Institut québécois de recherche sur la culture et Presses universitaires de Lyon, 1985), 587-603; F. Descarries-Bélanger, L'école rose... et les cols roses (Montréal, Éditions Albert StMartin, 1982). 


\section{CADRE D'ANALYSE: MARCHÉS FERMÉS ET FRONTIÈRES INTERPROFESSIONNELLES}

Notre objectif de recherche peut se traduire par un double questionnement. Premièrement, comment des professions telles que la diététique et la physiothérapie se situent-elles par rapport à ces autres professions qui partagent avec elles un même champ de savoir et de pratique et, par conséquent, une même clientèle potentielle? Deuxièmement, par quelles stratégies ces deux professions chercheront-elles à établir leur légitimité et à défendre leur statut dans un contexte concurrentiel? Plutôt que de recourir à des interprétations qui se réfèrent à des cadres sociaux globaux ${ }^{3}$, nous privilégierons les problématiques qui nous permettront de répondre de façon plus précise à ces questions. Dans cette perspective, la notion de "clôture sociale» (social closure) s'avère très importante. Empruntée à la théorie weberienne des rapports sociaux et développée surtout par F. Parkin, cette notion désigne les processus par lesquels des groupes sociaux visent à établir leur monopole ou à défendre leurs privilèges en élaborant des stratégies d'exclusion à l'endroit des groupes concurrentiels ${ }^{4}$. Cette approche a été utilisée par certains sociologues pour comprendre comment les professions réussissent à établir ce que M. Sarfati-Larson a désigné comme des «marchés protégés», C. Paradeise des «marchés de travail fermés» et E. Friedson des «chasses-gardées ${ }^{5}$ ».

Qu'elles se situent dans une perspective marxiste ou weberienne, les études concernées concluent que toute forme de clôture sociale débouche sur l'établissement de formes variées de monopoles professionnels ${ }^{6}$. Enfin, certaines problématiques mettent l'accent sur le fait que la clôture sociale s'appuie sur la légitimation que représente pour

3. En ce qui concerne la prolétarisation des professionnels liée au développement du mode de production capitaliste, voir H. Braverman, Travail et capitalisme monopoliste (Paris, Maspero, 1976); M. Sarfati-Larson, The Rise of Professionalism (University of California Press, 1977); M. Oppenheimer, "The Proletarianization of the Professional», P. Halmos, ed., Professionalization and Social Change (Grande-Bretagne, University of Keele, 1973), 213-228. Quant à la bureaucratisation des professions liée au contrôle accru de l'État, voir M. SarfatiLarson, op. cit; T. J. Johnson, «The State and the Professions: Peculiarities of the British», A. Giddens et G. Mackenzie, eds., Social Class and the Division of Labour (Cambridge, Cambridge University Press, 1982), 186-208; G. Dussault, «Professionnalisation et dé-professionnalisation», op. cit., 605-616; M. Renaud, «Les réformes québécoises de la santé ou les aventures d'un État 'narcissique'», L. Bozzini et al., Médecine et société. Les années 80 (Montréal, Éditions Albert St-Martin, 1981), 513-544.

4. F. Parkin, «Strategies of Social Closure in Class Formation», F. Parkin, ed., The Social Analysis of Class Structure (London, Tavistock, 1974).

5. M. Sarfati-Larson, op. cit. C. Paradeise, «Les professions comme marchés du travail fermés", Sociologie et sociétés, 20,2 (octobre 1988): 9-21. E. Friedson, Profession of Medicine. A Study of the Sociology of Applied Knowledge (New York, Dodd, Mead \& Co., 1972).

6. Nous avons pu constater, dans des analyses antérieures, que les privilèges liés à la clôture sociale s'exerçaient toutefois à des degrés bien différents selon que les groupes 
les professions l'institutionnalisation de leur savoir; que cette reconnaissance institutionnelle s'appuie sur l'État ou sur les instances de haut savoir?.

Malgré le fait que les professions tendent à se développer dans une logique de marché fermé et à ériger des barrières protectrices, il n'en demeure pas moins, soulignent la majorité de ces auteurs, que ces barrières restent précaires. Il faut se rappeler, en effet, que les professions sont des construits historiques qui s'inscrivent dans des rapports sociaux mouvants. Parmi les éléments qui contribuent à la remise en question de leurs privilèges, il y a la concurrence que représente l'émergence des nouvelles professions qui se situent dans des champs de savoir et de pratique connexes.

Dans le cadre d'une problématique qui vise à rendre compte des rapports entre professions rivales, nous considérerons également les notions d'exclusion et de démarcation. Si les politiques d'exclusion cherchent à maintenir carrément hors du champ de pratique d'une profession un ou des groupes sociaux déterminés, les stratégies de démarcation opèrent, quant à elles, de façon plus détournée, sinon plus subtile. Elles sont généralement pratiquées par des professions en position de pouvoir qui veulent maintenir ou créer une distance reconnue entre elles-mêmes et des professions connexes au statut moins prestigieux. La démarcation constituerait en quelque sorte une ligne de sécurité à l'abri de laquelle une profession chercherait à protéger ses acquis. Mais si le jeu de la négociation accompagne souvent les politiques de démarcation, ce processus n'exclut pas la confrontation $^{8}$. En ce qui concerne la diététique et la physiothérapie, cependant, nous verrons à l'œuvre de multiples stratégies de démarcation plutôt que des politiques brutales d'exclusion.

Par ailleurs, les rapports sociaux de sexe demeurent très peu visibles dans l'ensemble des problématiques évoquées jusqu'ici. À cet égard, la thèse de $\mathrm{A}$. Witz s'avère particulièrement intéressante ${ }^{9}$. Se

professionnels avaient accès à un monopole de pratique (pratique exclusive) ou bien au seul monopole du titre (titre réservé). Voir à cet égard: N. Fahmy-Eid et A. Charles, «Raison d'état ou État du plus fort? La diététique et la physiothérapie en quête d'une pratique exclusive au Québec, 1950-1980", Histoire sociale, 26,51 (mai 1993): 95-113.

7. A. Abbott, The System of Professions (University of Chicago Press, 1988). Voir également C. Paradeise, op. cit. et E. Friedson, op. cit.

8. Selon E. Friedson, l'exclusion renvoie au contrôle interne d'une profession et constitue une forme d'auto-défense face à ce qui est considéré comme une atteinte possible au statut professionnel. La démarcation quant à elle renvoie à un contrôle interprofessionnel visant à maintenir des distances avec une profession connexe. G. Larkin va jusqu'à référer aux concepts de «occupational imperialism» lorsqu'il évoque les stratégies d'exclusion. Voir à cet égard Occupational Monopoly and Modern Medicine (London, Tavistock, 1983).

9. A. Witz, Professions and Patriarchy (Londres/New York, Routledge, 1990). 
rapportant aux notions d'exclusion et de démarcation, Witz analyse les stratégies mises au point au cours de l'histoire par certaines professions masculines à l'égard d'une possible intégration des femmes dans leurs rangs. Ces stratégies se traduisent soit par une nette exclusion, comme ce fut le cas des corporations médicales face aux femmes au $\mathrm{XIX}^{\mathrm{e}}$ siècle, soit encore par des formes diverses de démarcation, comme dans le cas des politiques adoptées par les médecins également à l'endroit des infirmières et des sages-femmes. De leur côté, les groupes exclus développent soit des stratégies d'inclusion pour forcer des champs de pratique interdits (les femmes en médecine par exemple), soit des stratégies d'appropriation visant certains actes professionnels exercés jusque-là par des hommes (les infirmières face aux médecins par exemple).

Tout en présentant un intérêt certain pour notre analyse, la problématique de A. Witz s'inscrit toutefois dans un cadre chronologique précis, celui d'un $\mathrm{XIX}^{\mathrm{e}}$ siècle témoin des premières tentatives des femmes pour rejoindre les rangs des professions. Nous formulons, quant à nous, l'hypothèse qu'au $\mathrm{XX}^{\mathrm{e}}$ siècle, le jeu de la concurrence professionnelle ne passe pas uniquement par un clivage entre les sexes. On se rend compte que ce clivage intègre en fait des formes de clôture sociale où la démarcation peut aussi bien intervenir entre des groupes professionnels majoritairement féminins. Ce sera, comme nous le verrons, le cas des diététistes et des physiothérapeutes face aux techniques auxiliaires issues de leurs propres champs.

Il nous restera à voir à l'œuvre les éléments centraux de la problématique exposée ci-haut à travers les multiples problèmes de frontières auxquels font face la diététique et la physiothérapie au Québec entre 1950 et $1980^{10}$.

\section{1 - LES FRONTIÈRES AVEC LA MÉDECINE}

Toutes les professions paramédicales, la diététique et la physiothérapie comme les autres, connaîtront, bien qu'à des degrés divers, des problèmes de frontières interprofessionnelles avec la médecine. Le champ d'exercice de cette dernière s'avère en effet considérable, du moins si l'on se réfère à la description qu'en donne une étude commandée par la Commission d'enquête sur la santé et le bien-être social:

Il semblerait que l'acte médical est censé inclure tous les domaines des diverses professions paramédicales autres que la

10. Certains problèmes relatifs à l'accessibilité des sources récentes nous ont amenées à limiter notre recherche au début des années 1980. 
pharmacie et qui en constituent en quelque sorte des démembrements. Les médecins peuvent exercer tout l'éventail des actes médicaux ou paramédicaux. D'autre part, les praticiens des diverses professions paramédicales sont limités à leur spécialité particulière. Sauf pour la pharmacie et la chirurgie dentaire, l'on peut dire que le législateur a créé ou ratifié une hiérarchie juridictionnelle: les médecins, sous réserve des restrictions que nous avons indiquées ou de celles qu'ils s'imposent eux-mêmes, peuvent exercer dans n'importe quel domaine relevant du diagnostic ou du traitement y compris ceux qui intéressent d'autres professions. Il n'y a peut-être pas de subordination juridique entre ces corporations professionnelles et le Collège des médecins mais on constate une hiérarchie dans l'ampleur des juridictions ${ }^{11}$.

On peut comprendre qu'une définition aussi large du champ de pratique médical, assortie d'une perspective nettement hiérarchique des rapports interprofessionnels, puisse donner lieu à des rapports conflictuels entre les médecins et les professions paramédicales.

\section{A - Diététistes et médecins}

\section{Revendication d'un statut de collaboratrice}

Jusqu'à la fin des années 1950, les médecins semblent avoir favorisé le développement de la profession de diététiste en donnant leur appui autant à la création de départements et de programmes universitaires qu'à l'obtention du titre réservé ${ }^{12}$. Cette bienveillance, toute relative, s'amenuise toutefois avec les problèmes que posera la délimitation des frontières entre les champs de pratique des deux professions.

À partir des années 1960 , les diététistes reprochent, en effet, au corps médical son manque de collaboration et sa méconnaissance de leurs compétences: deux attitudes qui, selon elles, les empêchent d'être considérées comme des membres à part entière de l'équipe

11. C. A. Sheppard, «Les corporations professionnelles», L'organisation et la règlementation des professions de la santé et du bien-être au Québec, Annexe 12 de la Commission d'enquête sur la santé et le bien-être social (Québec, 1970), 2,2: 344.

12. C'est un médecin qui favorise en 1919 la mise sur pied d'un département de diététique au Montreal General Hospital (Corporation des diététistes du Québec, Annual Report 1968-1969 and History of the Corporation, 2). De plus, le baccalauréat en diététique de l'Université de Montréal est créé en 1942 à la suite d'une recommandation de la Faculté de médecine (Archives de l'Université de Montréal, Fonds du Secrétariat général, D35/679). Enfin, le projet de loi présenté par les diététistes en 1956 pour l'obtention du titre réservé reçoit l'appui de médecins provenant à la fois du milieu hospitalier et de différentes instances gouvernementales (Archives de l'Institut de diététique de l'Université de Montréal, dossier «Bill 232, Loi des diététistes; Correspondance 1956»). 
médicale ${ }^{13}$. On peut s'interroger sur les effets de ces protestations dans la mesure où l'on retrouve, encore en 1975, un témoignage qui exprime les mêmes griefs: les médecins considèrent les diététistes comme des exécutantes et leur laissent la portion congrue du travail d'équipe, alors qu'elles voudraient «vivre l'expérience de travailler avec les médecins et non pas pour les médecins ${ }^{14} »$.

Cette affirmation d'autonomie peut s'expliquer en partie par l'histoire de la diététique qui tire son origine de l'enseignement ménager, un champ de savoir totalement étranger à la médecine. Dès l'émergence de leur profession, les diététistes se considèrent donc, en vertu de leurs compétences spécifiques, comme des collaboratrices des médecins plutôt que comme des auxiliaires sous tutelle. Et depuis les années 1960, elles aspirent à limiter leur statut d'assistantes au cadre des traitements thérapeutiques.

Par ailleurs, l'insistance mise à se faire reconnaître comme partie intégrante de «l'équipe médicale» n'est sans doute pas fortuite. Cette notion d'équipe correspond à une nouvelle organisation du travail qui se généralise dans les hôpitaux entre 1955 et 1980 et coïncide avec l'essor des professions paramédicales ${ }^{15}$. Elle implique que, en vertu de leur savoir spécifique, plusieurs catégories de spécialistes aient leur place dans le processus de soins. On peut alors supposer que ce nouveau modèle de collaboration constitue pour de jeunes professions une porte d'entrée privilégiée dans un monde hospitalier où régnaient presque exclusivement jusqu'ici, bien qu'avec une autorité inégale, médecins et infirmières. Dans cette perspective, on comprend mieux l'insistance des diététistes à se faire reconnaître par les médecins comme de véritables membres de l'équipe médicale. Cependant, leurs revendications conserveront un ton relativement modéré. Elles éviteront d'attaquer directement les privilèges du corps médical sur la place publique et opteront plutôt pour des tactiques consistant à s'approprier certains actes jusque-là réservés aux médecins.

13. À cet égard, les témoignages qu'on retrouve dans les documents suivants sont éloquents: Corporation des diététistes, Mémoire à la Commission d'enquête sur la santé et le bien-être social (1967); "Journée d'étude», Bulletin de la Corporation des diététistes, 8,4 (février 1968): 5; L. Mercier, «À qui la faute?», L'hôpital d'aujourd'hui, 15,10 (octobre 1969): 50; «Rapport du Comité des relations professionnelles», Rapport annuel de la Corporation des diététistes du Québec (1970-1971): 16.

14. «Lettre ouverte», Bulletin de la Corporation professionnelle des diététistes du Québec (dorénavant CPDQ ou CDQ) (avril 1975): 20.

15. Le contexte historique dans lequel se développe le concept d'équipe médicale a été exposé par A. Petitat. Ce dernier ne l'étudie cependant qu'en ce qui concerne les médecins, les infirmières et les infirmières auxiliaires. Voir A. Petitat, Les infirmières. De la vocation à la profession (Montréal, Boréal, 1989), 156-169. 
Une stratégie d'appropriation: la prescription médicale liée au diagnostic

Les diététistes tenteront d'élargir leur champ de pratique pour y inclure, en particulier, un droit de regard sur la prescription médicale. Légalement, elles ne peuvent poser d'acte thérapeutique sans détenir une prescription à cette fin puisque seuls les médecins sont habilités par la loi à établir un diagnostic et à prescrire un traitement. Mais dès les années 1950, certaines diététistes affirment être suffisamment compétentes pour fonctionner à partir d'un plan de traitement au lieu d'une prescription détaillée ${ }^{16}$. En 1969, l'une d'entre elles va plus loin en affirmant que les membres de sa profession sont en mesure d'évaluer les fondements nutritionnels d'un état pathologique donné, ou même de corriger le contenu de certaines diètes prescrites:

On l'oublie [le fait que la diététicienne est une spécialiste de la nutrition] même au point qu'on ne la juge pas apte à réfuter certaines diètes erronées, basées sur des conceptions subjectives et arbitraires, et non sur des principes scientifiques. [...] La diététicienne est celle qui peut évaluer l'alimentation d'un malade, même de celui qui ne nécessite pas de régime spécial, d'en faire l'analyse et d'apporter les corrections qui s'imposent au niveau des habitudes alimentaires. Elle détermine aussi les causes d'un état nutritionnel déficient, en faisant une enquête alimentaire et un rapport détaillé des divers éléments nutritifs ${ }^{17}$.

Il s'agit là d'une opération discrète d'appropriation du droit de prescription, un acte réservé jusqu'ici aux seuls médecins. Sans contester officiellement ce monopole médical, les diététistes travailleront prudemment, mais assidûment, tout au long des années 1970 à repousser les limites qu'il leur impose. La citation suivante donne une bonne idée des précautions prises pour aborder ce terrain miné. En commission parlementaire, la représentante de la corporation répond ainsi à un médecin qui lui demande ce que deviendrait le pouvoir de prescription des médecins advenant le cas où les diététistes obtenaient un monopole sur leur champ de pratique:

16. Dès 1955 , la directrice de l'Institut de diététique de l'Université de Montréal décrit ainsi le rôle de la diététiste: «Du point de vue médical, la diététiste doit traduire en menus équilibrés, variés, adaptés au goût du malade des prescriptions diététiques en corrélation avec le diagnostic posé par le médecin». Voir R. Beaudouin, «La diététique», L'Hôpital d'aujourd'hui, 1,9 (septembre 1955): 59. Cinq ans plus tard, une diététiste affirme qu' «en prescrivant les diètes, soit générales soit spéciales, le médecin le fera en termes généraux, laissant le soin des détails à la diététiste ou à la surveillante de cuisine». Sœur Saint-Paul, «Le service alimentaire», L'Hôpital d'aujourd'hui, 6,5 (mai 1960): 44.

17. S. Mavrekakis, "La chaise à trois pattes», L'Hôpital d'aujourd'hui, 15,1 (janvier 1969): 15. 
Dans notre esprit ce pouvoir de prescription ne sera pas du tout limité. Au contraire, je pense l'avoir indiqué tout à l'heure, nous croyons que le traitement global reste la responsabilité du médecin. Alors, l'ensemble des prescriptions que nécessite un cas particulier relève de lui. S'il y a une prescription diététique qui est un des aspects de cette prescription, il reste quand même le premier responsable. Mais nous pensons qu'il peut difficilement, dans la plupart des cas, la faire de façon pleinement satisfaisante sans consultation avec le diététiste ${ }^{18}$.

Avec le temps, les diététistes indiqueront de plus en plus clairement leur intention de participer à l'établissement du plan de traitement. En 1978, un autre pas est franchi: le comité d'inspection professionnelle de la corporation considère que la diététiste-clinicienne «prescrit un traitement thérapeutique et établit un programme de traitement diététique personnalisé ${ }^{19}$ ».

Cette lutte que mènent les diététistes depuis les années 1950 s'appuie sur le seul argument qui puisse être jugé valable dans le monde professionnel: la maîtrise d'un savoir spécifique et spécialisé, dont même les médecins seraient dépourvus. Â cet égard, les diététistes jugent que les futurs médecins reçoivent une formation en nutrition inadéquate et que cette situation est responsable de nombreuses prescriptions incomplètes, irréalistes, «aberrantes, et le mot est choisi». Certaines demanderont même à quoi sert d'être des spécialistes «s'il faut courber l'échine devant le médecin dont le programme d'étude ne prévoit qu'un ou deux cours de nutrition et encore «à option ${ }^{20} \gg$ ?

Pour les diététistes, la solution à ce problème consiste à collaborer avec les médecins avant même qu'une diète ne soit prescrite. En 1977, le ministère des Affaires sociales paraît tenir compte de ces revendications et propose que l'enseignement des principes de base en nutrition devienne obligatoire dans toutes les facultés de médecine. En fait, il place les diététistes dans une position très inconfortable: elles ne peuvent logiquement s'opposer aux objectifs d'une telle politique mais s'inquiètent vivement de ses effets possibles:

18. Journal des débats, Commissions parlementaires, $3^{e}$ session, $29^{e}$ législature, $\mathrm{n}^{\circ} 99$ (12 octobre 1972): B-5994. Le souligné est de nous.

19. CPDQ, La Lettre, 3,6 (mars-avril-mai 1979): 8. Il est cependant précisé que la diététiste doit faire un rapport au médecin en cabinet privé.

20. Dès 1968, certaines diététistes soulignent que les prescriptions inadéquates sont relativements fréquentes. L. Pooler, «The Therapeutic Dietician», Bulletin de la CPDQ, 8,6 (octobre 1968): 9-12. Voir aussi: «Lettre ouverte», Bulletin de la CPDQ (avril 1975). La CDQ semble avoir la même opinion que ses membres sur ce point puisqu'elle juge en 1973 que «les médecins sont incapables d'enseigner l'application des régimes alimentaires» (CDQ, Procèsverbal, 20 juin 1973). 
Nous nous interrogeons sur le rôle que l'on semble vouloir confier au futur médecin. [...] Que signifie dans l'idée des auteurs l'expression «conseiller adéquatement ses clients»? Est-ce que dans l'avenir le médecin va se substituer au professionnel formé en diététique dans le but de guider l'homme dans son alimentation? Est-ce que «l'enseignement pratique en nutrition humaine» va rendre le futur médecin spécialiste dans ce domaine? Comment cet enseignement pratique se fera-t-il? Le but de l'enseignement de la nutrition aux professionnels ne serait-il pas plutôt de s'assurer le minimum de connaissances nécessaires au bon fonctionnement de l'équipe multidisciplinaire ${ }^{21}$ ?

On voit ici se profiler un réflexe de défense à l'égard du corps médical. Lorsqu'elles tentaient de s'approprier un droit de regard sur la prescription, les diététistes ne s'attaquaient qu'au monopole des médecins et non pas à leur droit de prescrire. Or, cette fois, il ne s'agit plus pour elles d'élargir leur sphère d'activité, mais bien de la protéger contre un groupe dont le prestige est supérieur au leur: accepter qu'on offre aux médecins une formation plus poussée en nutrition risquerait de porter atteinte à l'existence même de la diététique. Les diététistes préfèrent continuer à fonctionner au sein d'une équipe multidisciplinaire, quitte à poursuivre leur lutte pour collaborer à la prescription des traitements. Quitte aussi à continuer de «sensibiliser» - mais pas davantage - les internes en médecine à travers la formation qu'elles-mêmes leur donnent depuis longtemps ${ }^{22}$. Elles demeureraient ainsi les seules spécialistes de la nutrition - en attendant de voir plus tard le problème se poser autrement avec l'apparition des médecinsnutritionnistes.

Revendication d'une pratique privée

La pratique privée constitue un autre élément des visées autonomistes des diététistes face aux médecins. Au début des années 1970, ce type de pratique en est encore à un stade embryonnaire et la corporation commence tout juste à prendre les moyens pour la développer $^{23}$. La possibilité de nouveaux débouchés et l'accroissement de

21. «Rapport du Comité Ad Hoc du Département de diététique de l'Université Laval sur le document gouvernemental intitulé Une politique québécoise en matière de nutrition» (décembre 1977), 6.

22. CDQ, Mémoire soumis à la Commission d'enquête sur la santé et le bien-être social (1967). Institut de diététique et de nutrition de l'Université de Montréal, Projet d'un service de consultation en administration des services alimentaires hospitaliers (mars 1961).

23. En 1971, on ne dénombre que 10 diététistes en pratique privée, mais beaucoup de femmes semblent intéressées. Voir CPDQ, Procès-verbal, 18 mai 1971. La corporation imposera des exigences assez sévères pour déterminer celles qui seront habilitées à pratiquer en cabinet. 
la visibilité de ses membres ont certainement incité la corporation à favoriser ce type de pratique. Mais il est probable que l'autonomie professionnelle qui accompagne la consultation privée, et qui rappelle le modèle des professions libérales, a aussi été perçue comme un autre avantage appréciable.

Or, il est certain que plus la marge de manœuvre face à la prescription médicale sera grande, plus la pratique privée en diététique s'en trouvera facilitée. Prudente au tout début, la corporation s'engage graduellement dans cette voie. Forte de l'appui de principe donné par les associations de médecins et d'administrateurs d'hôpitaux, elle indique à ses membres, en 1972, qu'une prescription médicale n'est pas indispensable pour les clients qui ont besoin d'un régime alimentaire: il suffirait qu'un médecin recommande une consultation diététique. Subtile différence entre la prescription d'un régime par un médecin et la simple référence à une diététiste qui se charge ensuite de déterminer le traitement adéquat... ${ }^{24}$

Dès 1973 cependant, la corporation déplore que les médecins n'apportent pas leur entière collaboration à son projet: ces derniers préfèrent envoyer leurs patients dans les cliniques des hôpitaux ou se contentent de leur distribuer des copies de régimes standardisés, au lieu de les référer aux diététistes en pratique privée ${ }^{25}$. S'agirait-il d'une stratégie de démarcation des médecins à l'égard des diététistes? L'indépendance que confère la pratique privée inquièterait-elle de plus en plus un corps médical soucieux de maintenir son emprise sur ces professionnelles du paramédical ${ }^{26}$ ?

Il est en tout cas certain que, sur le terrain, les diététistes en pratique privée manifestent de plus en plus leur autonomie face aux médecins. Pour renforcer leur position, elles demandent en 1973 que leurs services soient couverts par l'assurance-maladie, ce qui leur assurerait une clientèle plus nombreuse puisque ces services deviendraient gratuits. L'État n'accède pas à cette demande, mais il leur accordera cependant un privilège important à la fin des années 1980: l'accès direct, c'est-à-dire la possibilité de traiter des patients sans qu'ils soient référés par un médecin. Les diététistes auront donc réussi à s'approprier une part du monopole des médecins sur le diagnostic en obtenant le droit de pratiquer sans prescription médicale.

24. CPDQ, Procès-verbal, 12 décembre 1972.

25. Selon la CPDQ, les cliniques externes des hôpitaux et les médecins constituent les principaux compétiteurs des diététistes en cabinet privé. Rapport annuel CPDQ (1972-1973), 23.

26. Il nous est difficile d'être très affirmatives étant donné que les sources exposant le point de vue des médecins sur cette question font cruellement défaut. 
Bien que problématiques, les relations entre diététistes et médecins semblent avoir été moins conflictuelles que celles de ces derniers avec d'autres professions paramédicales, comme la physiothérapie. Le fait que la diététique déborde largement le champ de la santé pourrait expliquer en partie cette situation. Si la majorité des diététistes pratiquent de plus en plus dans les hôpitaux ${ }^{27}$, une importante minorité d'entre elles travaillent dans d'autres secteurs: services alimentaires scolaires, ministères et services municipaux, CLSC et DSC, secteur commercial, éducation populaire, consommation, etc... Par ailleurs, même dans les hôpitaux, les diététistes n'assument pas uniquement des fonctions thérapeutiques. Elles dirigent les services alimentaires hospitaliers, ce qui comprend la gestion du personnel et des budgets affectés aux cafétérias et aux cuisines. Enfin, les personnes «traitées» par les diététistes ne sont pas nécessairement des malades:

Nous ne divisons pas les catégories d'individus en deux, quand nous pensons à l'acte diététique, les bien-portants et les malades. L'acte diététique reste le même, l'essence est la même. C'est qu'il s'applique à des besoins nutritionnels spéciaux et que ces besoins-là sont le lot des malades, c'est-à-dire des cas pathologiques, et le lot de conditions physiologiques parfaitement normales comme la grossesse et la croissance ${ }^{28}$.

Qui plus est, les diététistes s'attribuent, depuis les années 1960, un rôle important d'éducation auprès du public pour l'amener à adopter une alimentation équilibrée ${ }^{29}$; ceci à une époque où la prévention ne figure pas encore parmi les priorités d'un corps médical plutôt axé sur une pratique curative. C'est d'ailleurs sur ces deux derniers points que les diététistes misent, comme on l'a vu, pour contourner ou limiter la portée de la prescription médicale.

Enfin, il est possible que le lien établi à l'origine entre la diététique et l'enseignement ménager explique la relative indifférence avec laquelle les médecins semblent considérer le champ de l'alimentation humaine; indifférence dont les diététistes bénéficieront finalement jusqu'à un certain point. Le fait qu'elles aient réussi vers la fin des années 1950, sans coup férir semble-t-il, à dispenser elles-mêmes aux internes en médecine les cours relatifs à la nutrition en est une bonne illustration.

27. Les diététistes pratiquent en milieu hospitalier dans une proportion de $49 \%$ en 1959 , $73 \%$ en 1970 et $71 \%$ en 1975 . Chiffres compilés à partir de sources diverses de la CPDQ.

28. Journal des débats (12 octobre 1972): B-5989.

29 CDQ (Chapitre de Québec), Mémoire soumis aux autorités de l'Université Laval (mars 1965). CDQ, Mémoire soumis à la Commission d'enquête sur la santé et le bien-être social (1967). 
Tout ceci a probablement contribué à donner aux diététistes une certaine indépendance face aux médecins. Il n'en demeure pas moins que la dynamique propre aux relations interprofessionnelles, mélange d'appropriation et de démarcation, a joué d'une manière très tangible entre ces deux groupes.

\section{B - Physiothérapeutes et médecins}

\section{Deux définitions opposées de la physiothérapie}

On constate qu'à l'origine des problèmes de frontières entre la physiothérapie et la physiâtrie, il y a deux perspectives totalement opposées sur la nature du savoir physiothérapeutique et, conséquemment, sur la place et le rôle de la physiothérapie dans le domaine de la santé. Dès le milieu des années 1960, les physiâtres décrivent le physiothérapeute comme «un technicien qui applique certains traitements par les agents physiques, sur prescription médicale ${ }^{30}$ ». À travers cette définition très succincte, apparaît un objectif de démarcation qui se traduit par une double affirmation: premièrement, la physiothérapie se ramène à une technique et, deuxièmement, elle se limite à appliquer les directives liées au diagnostic établi par un médecin. Les physiâtres demeureront remarquablement fidèles à cette définition dont ils formulent une version presque identique cinq ans plus tard, devant la commission parlementaire chargée d'étudier «une législation gouvernant la physiothérapie». On peut lire dans leur mémoire que «le physiothérapeute reçoit une formation technique qui lui permet simplement d'appliquer un traitement prescrit par un médecin ${ }^{31}{ }^{\prime}$.

Face à une catégorisation qui ramène leur savoir spécialisé à un savoir-faire technique et surtout qui les confine à un rôle d'exécution, les physiothérapeutes opposeront à maintes reprises une perspective très différente. Dans le cadre de leur mémoire à la Commission Castonguay en 1968, elles prennent soin d'expliquer que leur profession présente la double caractéristique d'être à la fois une «science» et un «art». Elles affirment explicitement: «nous ne sommes pas des techniciens dans le sens technique du mot» pas plus d'ailleurs que

30. Association des physiâtres du Québec, Mémoire à la Commission CastonguayNepveu (septembre 1967), 5. Il est à noter que le mémoire des physiâtres parle des physiothérapeutes au masculin. Les documents produits par l'Association des physiothérapeutes procèdent parfois de la même façon. Nous utiliserons, quant à nous, le féminin étant donné que nous nous référons à une profession qui était et est encore majoritairement féminine.

31. Mémoire présenté à la Commission parlementaire par l'Association des physiâtres de la province de Québec en relation avec le bill 272 proposant une législation gouvernant l'exercice de la physiothérapie (février 1972), 8. 
«des opérateurs de machines ${ }^{32}$ ». Face à une commission où se joue en quelque sorte l'avenir de leur statut professionnel, non seulement les physiothérapeutes tiennent-elles à se démarquer des occupations sanitaires à caractère technique, mais elles rappellent, à plusieurs reprises, qu'elles partagent avec les physiâtres un champ de pratique commun dont elles n'entendent pas être exclues. Elles insistent particulièrement sur leur capacité de participer au diagnostic thérapeutique dans la mesure où «le physiothérapeute détermine la gravité d'une lésion nerveuse et la force musculaire, mesure le mouvement des articulations et l'expansion du thorax». On lit également dans leur mémoire que le physiothérapeute «doit être capable de participer à la recherche médicale, d'organiser et de surveiller la marche d'un département..., d'élaborer un programme d'entraînement clinique approprié et de le mettre à exécution».

Le rappel des attributions de la physiothérapie relativement à la participation au diagnostic et à l'élaboration d'un nouveau savoir constitue ce que nous avions défini au départ comme une stratégie d'appropriation par une profession donnée d'actes traditionnellement réservés à une profession concurrente au statut plus reconnu. On constate qu'il y a bien peu de ressemblance entre cette vision des choses et celle qui sous-tend la définition de la physiothérapie proposée par les physiâtres. Il n'est donc pas étonnant qu'une opposition aussi radicale au plan des idées ait débouché sur une opposition du même ordre dans le domaine de la pratique.

\section{Dénonciation d'une collusion entre les physiâtres et l'État}

$\mathrm{Au}$ cours de la décennie qui suit l'adoption du Code des professions en 1972, les physiothérapeutes mèneront un dur combat pour protéger leur champ de pratique contre ce qu'elles perçoivent comme un contrôle abusif exercé par les physiâtres sur la pratique de la physiothérapie. Mais le problème apparaît d'autant plus difficile à résoudre que les physiâtres chevauchent souvent, sur le plan professionnel, le double statut de voisins et de patrons, en particulier dans le cadre des institutions hospitalières. Logés en quelque sorte sur le même palier que les physiothérapeutes, mais occupant ainsi l'étage au-dessus, les physiâtres représentent, dans les faits, des concurrents de taille. De plus, quoique nouvellement venus dans le champ des spécialisations médicales ${ }^{33}$, ils bénéficient d'une clôture sociale effi-

32. Les physiothérapeutes de la Province de Québec Inc., Mémoire à la Commission d'enquête sur la santé et le bien-être social, (Commission Castonguay) (avril 1968), 4.

33. La physiâtrie n'est reconnue officiellement comme spécialité médicale que dans les années 1940. Jusqu'en 1955 encore, l'ensemble du Canada ne comptait que dix-huit spécialistes en médecine physique. Voir Journal of Canadian Physiotherapy Association, 7,2 (septembre 1955): 7 . 
cace sur le plan juridique. Ainsi, en plus du prestige et du statut social liés à la profession médicale, ils peuvent compter sur l'atout majeur que représente la reconnaissance légale par l'État (depuis 1847) de leur droit à l'exclusivité de leur pratique.

Quant aux physiothérapeutes, elles constateront assez vite que, dans les conflits qui les opposent aux physiâtres dès le début des années 1970, le titre réservé qui leur a été octroyé en 1972 ne constitue pas un bouclier bien solide, et encore moins une arme de combat efficace $^{34}$. Leurs multiples recours à l'arbitrage de l'État les confirmera rapidement dans l'idée que leurs rivaux bénéficient d'une certaine indulgence de la part des instances gouvernementales sinon d'une véritable connivence - à laquelle elles-mêmes n'ont pas droit.

À partir d'un tableau chronologique constituant en fait un réquisitoire violent contre les physiâtres, la Corporation professionnelle des physiothérapeutes (CPPQ) dressera, en 1976, un bilan amer des politiques gouvernementales qualifiées de partisanes ${ }^{35}$. On y rappelle qu'en 1967, la loi de l'assurance-hospitalisation autorisait la couverture des traitements physiothérapeutiques uniquement en milieu hospitalier, mais que, trois ans plus tard, l'État étendra cette couverture à la pratique en cabinet privé, à la condition toutefois que les traitements physiques - appelés en l'occurrence "physiâtriques» soient dispensés par un médecin spécialiste. Cependant, selon les physiothérapeutes, comme dans les faits aucun médecin n'effectue luimême ce type d'acte, cette entente «a permis à un petit groupe de médecins spécialistes (plus spécifiquement les physiâtres) de réclamer des honoraires professionnels pour des actes qui sont posés par les physiothérapeutes ${ }^{36}{ }$. Il s'agit, affirme la corporation, d'un contrôle à peine déguisé sur un champ de pratique où les physiâtres n'exercent souvent aucune pratique réelle. De plus, selon les auteurs du document, ceci représente ni plus ni moins qu'un coup de force organisé «aux dépens des physiothérapeutes et de la population ${ }^{37}$ ». La protection du public représente, en effet, une constante de l'argumentation

34. Voir à cet égard, N. Fahmy-Eid et A. Charles, «Raison d'État...», op. cit.

35. Corporation professionnelle des physiothérapeutes du Québec (CPPQ), Calendrier des événements relatifs aux irrégularités dans la couverture de la physiothérapie par l'État. Il s'agit d'un document annexé au rapport annuel de la CPPQ de 1976. (Á l'avenir, Calendrier des événements...)

36. Ibid., 60.

37. Ibid. Voir également le mémoire de la CPPQ au ministère des Affaires sociales, intitulé Rapport préliminaire de la CPPQ sur les éléments de problématique de la physiothérapie au Québec (1976), 14. Les physiothérapeutes y affirment que si l'État accordait à leur profession la pratique exclusive, «les actes thérapeutiques seraient bien définis et assureraient un minimum de protection à la population». 
des physiothérapeutes dans ce litige, et c'est le public qui est identifié comme le grand perdant des stratégies élaborées par les physiâtres pour courtcircuiter le recours aux services physiothérapeutiques.

Il est difficile d'évaluer la portée précise des revendications formulées par les physiothérapeutes, mais il reste, qu'en mai 1976, un arrêté en conseil annulera l'autorisation des paiements aux physiâtres pour des actes qu'ils n'ont pas posés eux-mêmes. Cependant, dans l'entente issue de cette décision gouvernementale, il semble que le flou entourant le sens de termes tels que «la présence» ou «la participation» (exigées) des physiâtres ait permis à ces derniers de contourner un règlement qui se voulait, au départ, plus contraignant à leur égard ${ }^{38}$.

De plus, la nouvelle entente précise que l'État ne remboursera pas les soins physiothérapeutiques en cabinet privé, alors que le coût d'actes similaires, pratiqués sous la surveillance - très lointaine diront les physiothérapeutes - d'un médecin spécialiste, sera assumé par la Régie de l'assurance-maladie. Les physiothérapeutes qualifieront ironiquement ce type de fonctionnement de «remote control». Enfin, avantage supplémentaire reconnu aux physiâtres, l'entente reconnaît à ces derniers un rôle d'intermédiaires obligatoires entre le médecin généraliste et la physiothérapeute. L'État, encore une fois, avait apparemment reculé devant le lobby médical. Ainsi, malgré une volonté affirmée de gestion rationnelle du champ de la santé, cet Étatemployeur, mais aussi arbitre, est accusé de conjuguer de façon maladroite, ou même nettement partisane, des objectifs économiques avoués avec des compromis politiques plus difficilement avouables.

Cinq ans plus tard, la corporation rappellera à nouveau dans un document de travail que, dans leurs litiges avec les physiâtres, ses membres n'ont bénéficié le plus souvent que d'un appui mitigé de la part de l'État ${ }^{39}$. Elle signalera également avec amertume la présence des médecins dans plusieurs instances publiques du champ de la santé et leur lobby bien plus efficace que celui des physiothérapeutes. Enfin, elle soulignera qu'en tant que groupe professionnel à forte majorité féminine, engagé dans un conflit avec des médecins spécialistes masculins, elle fait face à une forme de discrimination qu'elle n'hésite pas à qualifier de sexiste.

38. Calendrier des événements..., 64-65.

39. CPPQ, Réflexions de la Corporation professionnelle des physiothérapeutes du Québec sur le titre réservé (1981). 


\section{Dénonciation d'une délégation d'actes détournée de ses fins}

Parmi les problèmes les plus ardus que vivent les physiothérapeutes dans leurs relations avec les physiâtres au cours des années 1970, il y a celui du rapport triangulaire entre ces deux professions et celle des techniciennes en réadaptation physique (TRP). Le conflit généré par ce rapport peut se résumer ainsi: les physiâtres sont accusés de recourir systématiquement aux services des TRP plutôt qu'à celui des physiothérapeutes parce que cette collaboration leur évite des problèmes de partage de champs de juridiction et que, de surcroît, elle s'avère plus rentable sur le plan économique ${ }^{40}$. Cette pratique est surtout courante, semble-t-il, dans les cabinets privés que possèdent ou dirigent des physiâtres. Elle traduit, dans les faits, la perception qu'ont les physiâtres de la physiothérapie, définie par eux comme un champ de connaissances strictement techniques. Dans cette perspective, leur recours aux TRP constitue l'illustration d'une stratégie de démarcation à l'endroit des physiothérapeutes.

Le contentieux revêt un caractère particulièrement inquiétant au printemps 1977, alors que la corporation dénonce une vague de congédiements de physiothérapeutes employées dans les cabinets privés de physiâtres ${ }^{41}$. Non seulement la médiation de la Corporation professionnelle des médecins du Québec dans ce dossier ne semble pas avoir porté fruit, mais elle a failli détériorer le climat plus serein qui avait caractérisé jusque-là les rapports entre les physiothérapeutes et les omnipraticiens ${ }^{42}$.

La question du remplacement des physiothérapeutes par des TRP restera à l'ordre du jour du contentieux avec les médecins spécialistes tout au long des années $1970^{43}$. Les répercussions sur la pratique des physiothérapeutes apparaissent d'autant plus importantes que, malgré les multiples représentations de la CPPQ, la Régie d'assurance-maladie du Québec continuera à défrayer les coûts des actes physiâtriques dans les cliniques privées tenues par les physiâtres, mais non les actes physiothérapeutiques dans celles tenues par les physiothérapeutes ${ }^{44}$. On peut imaginer que, dans ces conditions, ces derniers établissements auront beaucoup de mal à concurrencer leurs rivaux.

40. Calendrier des événements...

41. CPPQ, «Document», $2^{e}$ Bureau, document $n^{\circ} 104$. Lettre au président de la Corporation professionnelle des médecins du Québec (CPMQ), mai 1977.

42. CPPQ, Procès-verbal, 7 mars 1978.

43. La question du recours des physiâtres aux TRP ne trouvera en fait un élément de solution que lorsque les physiothérapeutes obtiendront, à la fin des années 1980 , le droit de traiter aussi bien les patients référés par des médecins que ceux qui n'ont pas eu recours à cette étape préliminaire. C'est ce qu'on désignera par le droit à l'accès direct.

44. Voir l'éditorial intitulé «L'acte physiothérapeutique, une réalité», Physio Québec, 5,4 (décembre 1980). 
Au cours de cette même décennie, les médecins tenteront de régler à leur avantage leur conflit avec les physiothérapeutes en présentant à l'Office des professions du Québec un projet de délégation d'actes médicaux aux TRP. Cette guérilla juridique amènera les physiothérapeutes à présenter à leur tour aux instances concernées une argumentation élaborée sur les dangers de la stratégie adoptée par les médecins. Le mémoire soumis par la CPPQ rappelle les objectifs initiaux assignés par le législateur à la délégation d'actes:

Tel que originellement conçu, le mécanisme de la délégation des actes, dans le domaine de la médecine en particulier, devait permettre d'assouplir la rigidité de ce champ d'exercice exclusif et de régulariser en droit une situation de fait, en autorisant certaines classes de personnes, autres que les médecins, à poser certains actes traditionnellement réservés à ceux-ci ${ }^{45}$.

Or, soutient la CPPQ, le projet de loi des médecins non seulement opère «un glissement par rapport à l'objectif initial de la délégation des actes», mais constitue «une véritable tentative de détournement de l'ensemble du mécanisme». En fait, au lieu d'assurer une plus grande flexibilité du champ d'exercice exclusif attribué aux médecins, un tel projet se trouve à étendre implicitement les limites de ce champ en y incluant des actes qui appartiennent en propre aux physiothérapeutes, en plus d'autres qui «peuvent même être posés par des personnes qui ne sont ni physiothérapeutes, ni techniciens». De plus, conclut la $\mathrm{CPPQ}$, ce projet qui sert uniquement des intérêts corporatistes constitue une forme légale d'usurpation ${ }^{46}$.

Pour contrer ce qu'elle perçoit comme une politique impérialiste, la CPPQ cherchera des solutions visant à affranchir ses membres de la tutelle médicale et à leur assurer une plus grande autonomie.

\section{Revendication d'une plus grande autonomie professionnelle}

Parmi les solutions visant à atteindre cet objectif, l'obtention du droit à l'établissement du plan de traitement du malade apparaît aux physiothérapeutes comme primordial. Tenant compte des inquiétudes exprimées à maintes reprises par le corps médical sur les effets présumément négatifs liés à l'exercice d'un tel droit, les physiothéra-

45. Mémoire de la CPPQ concernant le projet de délégation d'actes médicaux aux techniciens en réadaptation physique par les médecins (mars 1984): 3.

46. Ibid., 3-7. Le problème auquel font référence les physiothérapeutes tient probablement au fait que le champ de pratique des médecins ne semble pas avoir de balises légales très précises et peut ainsi, en principe, couvrir l'ensemble des actes médicaux de toutes catégories, $y$ compris les actes physiothérapeutiques. 
peutes tiendront constamment à ce sujet un discours conciliant. Il n'est nullement question, affirment-elles, de courtcircuiter la prescription médicale et donc de remettre en cause le diagnostic qui la sous-tend. Il s'agit, par contre, de revendiquer une forme d'expertise que le niveau de formation théorique et pratique des physiothérapeutes les habilite parfaitement à exercer ${ }^{47}$.

À cet égard, la position des physiâtres restera inchangée. On a vu que, à leurs yeux, les physiothérapeutes n'ont accès qu'à un savoir technique qui devrait les confiner à un simple rôle d'exécution. Dans le cadre d'une commission parlementaire ils affirment, déjà en 1972 , que la formation de la physiothérapeute ne lui permet en aucun cas «d'arrêter un plan de traitement» et ils font leurs les conclusions d'un juriste européen affirmant qu' «en aucun cas l'auxiliaire [qui désigne ici la physiothérapeute] n'est maître de la progression du traitement, de ses modifications... ${ }^{48}$ ». Pour les physiâtres, il s'agit manifestement de se démarquer des physiothérapeutes, de protéger leur propre champ de juridiction de toute forme d'appropriation, et d'assurer ainsi à leur profession une clôture sociale étanche. La corporation des médecins, de son côté, conseille aux physiothérapeutes qui jugent une ordonnance inadéquate de référer le problème au médecin traitant afin que ce dernier réévalue la situation et établisse une nouvelle prescription ${ }^{49}$.

Jusqu'à la fin des années 1970 , les positions des deux corporations varieront très peu, mais vers la fin de la décennie, on note un ton plus conciliant chez les physiothérapeutes lorsqu'elles abordent la question du plan de traitement ${ }^{50}$. Leur lutte pour une autonomie accrue a risqué toutefois d'être freinée par le jugement déclaratoire rendu en 1981 par la Cour supérieure en faveur des physiâtres dans le conflit qui opposait, depuis quelques années déjà, ces derniers aux physiothérapeutes de l'Institut de réadaptation de Montréal $^{51}$. Le jugement stipulait, en effet, que les physiothérapeutes n'étaient en aucune façon habilitées à

47. «The Changing Role of the Physical Therapist in Canada», Journal of Canadian Physiotherapy Association, 22,5 (décembre 1970). Voir également Mémoire de la CPPQ au ministere des Affaires sociales (1975).

48. Mémoire présenté à la Commission parlementaire par l'Association des physiâtres...,

49. CPPQ, Procès-verbal, 31 octobre 1977. Ce procès-verbal donne le compte rendu d'une rencontre entre la CPPQ et la corporation des médecins.

50. En avril 1979, le président de la CPPQ fait état de ses réflexions dans un document intitulé «Quelques opinions sur les attentes des physiothérapeutes envers les médecins» où il réitère les positions de la Corporation au sujet du plan de traitement des malades tout en affirmant que les physiothérapeutes reconnaissent la compétence des médecins et «entendent poursuivre leurs efforts de communications».

51. Jugement déclaratoire $n^{\circ} 500$ 05-007849-803 rendu par la Cour supérieure en date du 6 août 1981 . 
modifier «de leur chef» les prescriptions formulées par le médecin spécialiste. Le même jugement leur a néanmoins reconnu le droit d'établir, à la suite de «questions, tests et examens», un plan de traitement et de l'appliquer ${ }^{52}$. Aussi, malgré les limites qui leur sont imposées en ce qui a trait à la modification de la prescription médicale, il reste que la reconnaissance formelle du droit à l'établissement d'un plan de traitement a constitué pour les physiothérapeutes une étape décisive dans leur lutte pour une autonomie professionnelle accrue.

\section{2 - LES FRONTIÈRES AVEC LES TECHNIQUES AFFILIÉES}

\section{A - Diététistes et techniciennes en diététique}

Le premier programme de techniques de diététique s'ouvre en 1969 au niveau du cégep et sanctionne l'arrivée d'une nouvelle occupation dans le champ de la nutrition ${ }^{53}$. L'apparition des techniciennes ne se fait cependant pas contre le gré des diététistes qui l'envisagent depuis 1959 et le demandent formellement à l'État en $1967^{54}$. A cette époque d'ailleurs, la corporation songe à favoriser la structuration, sous sa supervision, non pas d'un seul mais de deux groupes occupationnels: des techniciennes initiées aux principes élémentaires de la nutrition et un personnel auxiliaire formé au secondaire pour des postes tels que «serveuse de cafétéria» et «aidecuisinier». Un lien peut sûrement être tracé entre cette volonté de créer deux occupations auxiliaires et l'emphase que les diététistes mettent, tout au long de la période 1940-1980, sur l'importance du rôle de la diététiste-chef dans la gestion des services alimentaires hospitaliers. Dans cette perspective, cette dernière doit coordonner des tâches relevant à la fois de la diététique (diétothérapie, enseignement, recherche) et de la production ou de la distribution alimentaires (cuisines et cafétérias). Ainsi définie, cette fonction comporte donc une part importante de gestion du personnel, qualifié et non qualifié.

Jusqu'au milieu des années 1970, la corporation ne semble pas trop redouter l'apparition des techniciennes, persuadée de pouvoir maintenir ces dernières à l'intérieur des limites qu'elle leur a imparties et de conserver ainsi le contrôle du champ. Dans cette optique, on envisage en 1974 de faciliter aux techniciennes l'accès à l'université et d'élargir l'éventail de leurs fonctions en leur déléguant à l'avenir

52. Ibid., 4.

53. Avant l'arrivée de ces techniciennes, les diététistes se faisaient seconder, comme nous le verrons plus loin, par des «auxiliaires en alimentation» formées dans les instituts familiaux. (1967).

54. CDQ, Mémoire soumis à la Commission d'enquête sur la santé et le bien-être social 
«de plus en plus de tâches y compris des tâches de contrôle et des tâches de transmission de connaissances élémentaires ${ }^{55}$ ». On veut ainsi leur ouvrir - dans une certaine mesure - la plupart des secteurs où travaillent les diététistes: contrôle de l'approvisionnement et de la distribution, supervision et formation du personnel auxiliaire, participation aux traitements et aux recherches sous supervision d'une diététiste. La Corporation professionnelle des diététistes du Québec (CPDQ) exprime en ces termes l'avantage que représente pour ses membres la constitution d'un groupe de techniciennes:

Le technicien en diététique est né. Il faut lui donner la place qui lui revient, que nous lui avons préparée. Il apportera une aide précieuse et rehaussera le niveau de notre profession en accomplissant les tâches techniques qui nous ont trop souvent accaparés, augmentant ainsi notre efficacité au travail ${ }^{56}$.

Les techniciennes en diététique revêtent un intérêt supplémentaire aux yeux de la corporation: en déchargeant les diététistes d'un certain nombre de tâches, elles leur permettent de pallier à la pénurie que l'on déplore depuis longtemps dans la profession et d'assurer des services de nutrition sur une plus grande échelle. C'est l'argument que fera valoir - sans succès d'ailleurs - la corporation dans ses démarches en 1971 pour que l'État accorde exclusivement à ses membres le privilège de pratiquer la diététique ${ }^{57}$.

Si la corporation encourage dès le début l'entrée des techniciennes sur le marché du travail, elle n'accepte pas toutefois que ces dernières servent à remplacer les professionnelles formées à l'université. La diététique n'est pas totalement à l'abri d'un tel danger, puisqu'il avait fallu démontrer à deux reprises, dans les années 1960, la pertinence de conserver la formation au niveau universitaire ${ }^{58}$. La corporation s'assure donc d'être présente à toutes les discussions entourant la formation et les tâches des techniciennes. Elle participe avec le ministère de l'Éducation à l'élaboration des programmes qui seront mis sur pied dans les cégeps à partir de 1969, détermine les futures tâches des techniciennes et bâtit des organigrammes de services diététiques hos-

55. J. Sévigny, Opération Sciences de la santé. Document de travail. Sous-secteur diététique: perspectives d'avenir, Rapport effectué pour le Comité directeur de l'Opération Sciences de la santé (mai 1974).

56. Y. Prémont-Matsusaki (déléguée de la CDQ au Comité de programme), «Le technicien en diététique», Bulletin de la $C D Q, 2,4$ (décembre 1971): 7.

57. Journal des débats (12 octobre 1972): B-5994.

58. Cette démonstration a dû être faite en 1962 devant la Commission royale d'enquête sur l'enseignement (Commission Parent) et en 1969, devant le Comité d'étude des professions auxiliaires de la santé (Comité Bergeron). 
pitaliers indiquant la position hiérarchique respective des unes et des autres. Il est certain que la fonction de diététiste-chef dans les hôpitaux assure aux diététistes un poids suffisant pour leur permettre de tracer elles-mêmes la ligne qui sépare les deux champs de pratique.

Dès le début, les diététistes mettent donc en œuvre des stratégies de démarcation visant à protéger leur champ. Le fait de se réserver le secteur de la diétothérapie en est un exemple. Le soin avec lequel est discuté le titre porté par les techniciennes en est un autre. Dès 1971, la corporation s'oppose ainsi à ce que les «techniciennes en alimentation» au cégep deviennent des «techniciennes en diététique», et elle ne renonce qu'au dernier moment à envoyer une protestation au ministère de l'Éducation contre ce qu'elle considère comme une violation de son titre réservé: «Un tel nom ferait confondre dans l'idée du public les diététistes et les techniciens en diététique, les premiers ayant suffisamment de problèmes d'identification auprès des autorités hospitalières et du public en général, sans y apporter plus de confusion $^{59}$.»

Un tel débat, qui pourrait a priori sembler un peu futile, cache pourtant des enjeux importants. Les diététistes n'ont en effet aucun intérêt à ce que le public ou les administrations hospitalières les confondent avec les techniciennes; c'est pourquoi l'image de la profession et le titre porté par chacun des groupes prennent à leurs yeux une telle importance $^{60}$. En outre, il faut se rappeler que les diététistes n'ont obtenu de l'État que le titre réservé et non la pratique exclusive, ce qui les rend d'autant plus vulnérables à cet égard ${ }^{61}$.

Quelques années plus tard, les membres de la corporation cachent de moins en moins leur préoccupation au sujet de la formation que reçoivent les techniciennes; une formation souvent jugée équivalente à celle du baccalauréat universitaire en nutrition. Une situation jugée d'autant plus inquiétante qu'un certain nombre de diététistes n'ont pas suivi l'évolution de la profession, d'où une différence qui s'amenuise entre professionnelles et diplômées du cégep. D'autres diététistes s'alarment du fait que des techniciennes s'approprient des fonctions

59. CDQ, Procès-verbal, 29 juin 1971. La CDQ ne réussira pas à faire valoir son point de vue. Et en 1971, le titre du programme collégial «Alimentation» change pour celui de «Techniques de diététique» (Cahiers de l'enseignement collégial, 1969-1971).

60. Un sociologue de l'Office des professions affirme d'ailleurs en 1979 que «pour conquérir un statut professionnel particulier, les personnes exerçant une activité de travail doivent d'abord se donner une identité qui leur permet de se reconnaître entre elles et de se différencier des autres». R. Laliberté, «La professionnalisation des occupations, une tendance à accentuer ou à renverser?», Critère, 25 (printemps 1979): 27.

61. N. Fahmy-Eid et A. Charles, «Raison d'État ou État du plus fort?...», op. cit. 
d'enseignement de la nutrition et même de la diétothérapie ${ }^{62}$. En 1976, on avoue que des tensions existent entre les deux groupes, que «l'articulation entre techniciennes et diététistes n'est pas encore satisfaisante et que l'augmentation du nombre d'étudiantes en techniques diététiques n'arrangera pas les choses ${ }^{63}{ }^{\prime}$. Cette question du nombre élevé de techniciennes préoccupe les diététistes au point qu'elles demandent une limitation du nombre d'admissions dans cette branche.

Ce revirement peut avoir plusieurs raisons. Il est fort possible que, à l'usage, le contrôle des frontières interprofessionnelles par les diététistes se soit révélé plus ardu que prévu. Les techniciennes ellesmêmes ont peut-être aussi manifesté une volonté de desserrer le filet tissé autour de leur champ de pratique, un peu comme l'avaient déjà fait les infirmières auxiliaires une décennie plus tôt ${ }^{64}$. Enfin, il est manifeste que les techniciennes représentent une menace dans la mesure où leurs services reviennent moins cher à l'État et aux administrations hospitalières. Envisagés de manière sereine au début, les rapports avec les techniciennes se détériorent donc par la suite.

\section{B - Physiothérapeutes et techniciennes en réadaptation physique}

Les problèmes de frontières entre la physiothérapie et la technique en réadaptation physique remontent à la naissance même de cette dernière catégorie d'emploi qui voit le jour en 1969. Or, on peut dire que cette naissance fut, pour le moins, non désirée.

Pour les physiothérapeutes, il s'agit tout d'abord de protéger leur champ de pratique et, surtout, de maintenir au niveau universitaire un savoir qu'elles craignent voir se dévaluer. Aussi, devant la Commission Castonguay, en 1968, puis un an plus tard devant le Comité des professions auxiliaires de la santé, l'Association des physiothérapeutes se dit fermement opposée à la création d'une occupation à caractère technique, située dans le prolongement de la physiothérapie ${ }^{65}$. Si

62. Bulletin de la $C P D Q$, (rapport d'ateliers tenus le 5 juin) (novembre 1976). On y soulève autant le problème de la formation «trop poussée» des techniciennes que l'enseignement de la nutrition donné par ces dernières dans certains cas.

63. Opérations Sciences de la santé, 2 (1976), ministère de l'Éducation et ministère des Affaires sociales (Québec, 1976), 272. Dès 1972, pas moins de 804 étudiantes étaient inscrites en technique d'alimentation. Voir Bulletin CPDQ (automne-hiver 1976-1977).

64. D. Nepveu, Le mouvement de professionnalisation des infirmieres-auxiliaires au Québec, 1950-1974, mémoire de maîtrise (histoire), Université du Québec à Montréal, 1985.

65. En ce qui concerne les positions défendues devant la Commission Castonguay, voir Les Physiothérapeutes de la Province de Québec Inc., Mémoire d la commission..., et le procèsverbal de l'assemblée générale de l'association en date du 24 avril 1968. En ce qui concerne la perspective défendue devant le Comité Bergeron, voir Les Physiothérapeutes de la Province de Québec Inc., Mémoire au Comité d'étude sur les professions auxiliaires de la santé (1969). 
l'association n'a pu empêcher un tel projet d'être mené à terme en 1970 (le cours se donne dès l'automne 1970 au Cégep de Chicoutimi), elle tentera, par tous les moyens, d'en minimiser les «dégats» sur le plan du statut professionnel. Elle le fera surtout à partir d'une redéfinition précise de l'étendue du champ de juridiction et de pratique des physiothérapeutes par rapport à celui des TRP. Il s'agira, en fait, d'élaborer des stratégies de démarcation, et aussi de contrôle, par rapport à une occupation qu'il apparaît important de contenir à l'intérieur de frontières bien précises.

La démarcation avec les TRP semblera aux physiothérapeutes d'autant plus urgente à établir que, dès le départ, plusieurs instances du champ sanitaire semblent intéressées à abolir les frontières entre les deux groupes. Ainsi, dès 1972, soit à la veille de l'adoption du Code des professions, un Comité ad hoc formé de représentants des ministères de l'Éducation et des Affaires sociales ainsi que du réseau collégial et universitaire, recommande que les futures TRP diplômées des collèges soient traitées, sur le marché du travail, sur un pied d'égalité avec les physiothérapeutes puisqu'elles auront reçu une formation équivalente à celle de ces dernières. On rappelle, du même souffle, que le nouveau baccalauréat en physiothérapie - qui remplace désormais l'ancien diplôme de deux ans - sera orienté vers l'administration ou l'enseignement ${ }^{66}$. On se souvient qu'en 1972 également, les recommandations des physiâtres à la commission parlementaire chargée d'élaborer un code des professions allaient dans le même sens. Même si elles ne déboucheront pas nécessairement sur des actions concrètes, ces tentatives de limitation de leur formation seront perçues par les physiothérapeutes comme des stratégies d'appropriation de leur champ, ce qui les incitera à une vigilance accrue.

La Corporation visera, dans un premier temps, à départager de façon très précise les tâches dévolues aux physiothérapeutes et aux TRP. Un premier document de travail, produit en 1976, établit les niveaux de compétence de chacun des deux groupes, leurs champs d'action respectifs et va jusqu'à dresser une liste détaillée des actes qui devraient être réservés aux physiothérapeutes ${ }^{67}$. Deux ans plus tard, l'exécutif de la CPPQ sollicitera une réunion conjointe avec les ministères des Affaires sociales et de l'Éducation pour traiter de la "complémentarité de la formation des intervenants dans le domaine de la réadaptation physique... de la complémentarité des rôles, responsa-

66. Rapport du comité ad hoc sur l'intégration des techniciens en réadaptation au marché du travail (1972), 19.

67. Tâches du physiothérapeute. Document de travail (septembre 1976). 
bilités et fonctions de ces mêmes intervenants ${ }^{68}{ }$. Il $s$ 'agissait manifestement d'un dossier ardu qui donnera lieu à la rédaction d'un second document de travail dans la même ligne que celui de 1976, mais avec des précisions supplémentaires ${ }^{69}$.

À la fin des années 1970 , les problèmes de frontières interprofessionnelles ne sont pas encore résolus. Ils le sont d'autant moins que les TRP, de leur côté, proposent une définition de leur rôle qui ressemble étrangement à celle des physiothérapeutes. Ainsi, dans le cadre de leur congrès de 1978, le représentant du Cégep de Chicoutimi affirme:

[Le TRP] doit évaluer et traiter le patient passé par un thérapeute, fixer les objectifs de traitement, établir ce traitement et le modifier au besoin, assumer la responsabilité des soins en réadaptation... en collaboration avec le médecin traitant ou le physiothérapeute. Dans tous les domaines étudiés, le TRP doit être capable d'identifier les pathologies et les rééduquer ainsi que de les évaluer ${ }^{70}$ ».

En plus d'objectifs qui s'apparentent à ceux des physiothérapeutes, on note dans cette déclaration une volonté d'autonomie professionnelle analogue à celle que les physiothérapeutes n'ont cessé, pour leur part, d'affirmer face aux physiâtres. Cependant, la CPPQ déclarera à ses membres que «l'évaluation du plan de traitement et certains actes physiothérapeutiques plus complexes ne sauraient être délégués aux techniciens ${ }^{71}{ }$.

Au seuil des années 1980, l'impasse semble toujours totale et alimente un climat de mécontentement au sein des deux groupes ${ }^{72}$.

68. CPPQ, Procès-verbal, 17 mai 1978.

69. Définition des tâches du physiothérapeute, 22 août 1978. La même année le ministère du Travail confiera, de son côté, à la firme SORECOM, une enquête sur la définition des tâches des physiothérapeutes. C'était, pour le moins, confirmer que la division des rôles entre physiothérapeutes et TRP n'était pas un problème que la législation était véritablement parvenue à résoudre.

70. J. Pérard, «La formation académique du technicien en réadaptation physique», Rapport du congrès 1978, 4.

71. CPPQ, «Rapport du président», Rapport annuel 1978-79, 10.

72. Dans le cadre de deux enquêtes, menées en 1978 puis en 1980, l'Association professionnelle des techniciens en réadaptation physique du Québec (APTRPQ) pourra prendre la mesure du mécontentement exprimé par ses membres à l'endroit des physiothérapeutes. Voir à cet égard le Rapport du comité exécutif de l'APTRPQ (1978) et les résultats d'un questionnaire aux TRP (dossier «Congrès 1980»). Les conflits entourant les frontières interprofessionnelles apparaissent cependant moins difficiles à régler avec des professions pourtant proches de la physiothérapie comme l'ergothérapie ou l'inhalothérapie. En ce qui concerne les ergothérapeutes, les physiothérapeutes ont dû lutter lors de l'élaboration du Code des professions afin d'éviter une fusion des deux professions sur le plan légal (cf. Journal des débats, Commissions parlementaires (31 août 1972): B-5076 à B-5083). Avec les inhalothérapeutes, les problèmes 
Qu'il s'agisse de ses rapports avec les physiâtres ou avec les techniciennes en réadaptation physique (TRP), la physiothérapie a dû mener, depuis les années 1960 surtout, une série de batailles sur plusieurs fronts. Il est évident que les plus délicates d'entre elles demeurent celles qui les ont confrontées aux TRP. Non seulement cette dernière profession occupe-t-elle un champ de pratique proche de celui de la physiothérapie et ses frontières sont restées assez floues, mais ni l'usage que font les physiâtres de cette situation ambiguë, ni la protection mitigée du titre réservé accordé aux physiothérapeutes par l'État ne viennent atténuer les difficultés que suscite une telle conjoncture. Cette situation débouchera sur une volonté marquée de la physiothérapie de défendre fermement des frontières menacées à la fois par le haut (la médecine physiâtrique) et par le bas (les techniques affiliées).

\section{3 - LES FRONTIÈRES AVEC D'AUTRES GROUPES OCCUPATIONNELS}

\section{A - Diététistes et diplômées en sciences ménagères}

La diététique est née des sciences domestiques. Au fil du temps cependant, les diététistes tenteront de se distancer des spécialistes de l'enseignement ménager puis, graduellement, de les exclure de leur champ. Au début des années 1970, les jeux sont faits: les sciences domestiques ont plus ou moins disparu de l'horizon de la diététique, sauf peut-être en milieu scolaire. Les premiers indices de cette évolution se manifestent dès les années 1940, en particulier lors de la création de l'Institut de diététique de l'Université de Montréal:

Cette enquête [menée en 1938 par des médecins de l'Université de Montréal sur l'état de la formation en diététique] révéla vite que dans la Province de Québec l'enseignement de la nutrition, tel que donné dans les Écoles ménagères, ne pourrait jamais fournir à nos hôpitaux les diététistes compétents dont la Faculté de médecine rêvait pour les hôpitaux de cette Province. [...] Si, en terre québécoise, l'Institut de diététique et de nutrition a d'abord fait un peu figure d'intrus, il vient cependant s'ajouter à la longue liste d'instituts anglo-américains et anglo-canadiens ${ }^{73}$.

paraissent avoir été plus délicats et plus compliqués à résoudre (cf. Mémoire de la $C P P Q$ présenté à l'Office des professions en mars 1977). La CPPQ rappellera notamment que le travail des techniciens en inhalothérapie devra se faire sous le contrôle exclusif des physiothérapeutes. 73. R. Beaudouin, «Diététique et nutrition à la Faculté de Médecine de l'Université de Montréal», Union médicale du Canada, 75 (avril 1946): 1. 
On voit ici se profiler la nécessité pour les diététistes de justifier l'existence de leur profession. Et dans cette perspective, la pratique en milieu hospitalier constitue une caractéristique qui leur permet de se démarquer de l'enseignement ménager. La coupure ne se fait pas spontanément cependant puisque le même article précise que, outre une carrière de diététiste, «les divers postes d'enseignement ménager en général, enseignement, direction ou travail de publicité dans les sciences ménagères, tenue de maison, budget, couture, décoration intérieure» font partie des débouchés possibles pour les diplômées en diététique.

Les sources demeurent muettes sur le sujet pendant un certain temps, mais la question refait surface une vingtaine d'années plus tard. Les diététistes semblent encore ambivalentes face aux sciences domestiques. En 1960, leur association explique la pénurie de diététistes notamment par l'aspect beaucoup trop «ménager» du cursus universitaire, caractéristique qui éloigne les hommes de la profession ${ }^{74}$.

En 1962 pourtant, l'Association de diététique du Québec présente un mémoire conjoint avec la Montreal Home Economics Association à la Commission royale d'enquête sur l'enseignement. Les deux groupes déclarent que ni les sciences domestiques ni la diététique ne sont des techniques et que ces disciplines méritent une formation au niveau des trois cycles universitaires, puisque «la formation universitaire en sciences domestiques assure à la femme non seulement une éducation supérieure conforme à sa personnalité et à son rôle futur au foyer mais d'un point de vue plus largement social, elle la prépare à l'exercice de fonctions éminemment utiles à la collectivité ${ }^{75 n}$. Ces associations déplorent, en outre, que les sciences ménagères ne bénéficient pas d'une véritable reconnaissance universitaire en milieu francophone - exception faite de la diététique. On souligne leur confinement à des écoles ou à des instituts qui n'ont pas le statut de départements universitaires, le niveau peu élevé des programmes offerts, le manque de qualification du corps enseignant, l'impossibilité pour les diplômées d'entrer à l'université sans reprendre une partie de leurs études, etc...

Dans ce mémoire, il est intéressant de noter que la diététique est présentée comme faisant partie du même champ académique que l'enseignement ménager et que deux visions du rôle des femmes coexis-

74. Association de diététique du Québec, Le rôle et le statut des diététistes professionnelles dans les hôpitaux de la Province de Québec, Mémoire soumis au ministre de la Santé (mai 1960), 14.

75. Association des diététistes du Québec, Mémoire sur l'enseignement ménager présenté à la Commission royale d'enquête sur l'enseignement (juin 1962), 38. 
tent: celle de futures mères-épouses, mais aussi celle de professionnelles. On peut voir là une certaine reconnaissance par les diététistes de leur filiation avec les sciences domestiques. Mais cette reconnaissance s'évanouira rapidement au fur et à mesure que la formation académique des filles se verra assigner, au Québec, un objectif de rentabilité sur le marché du travail. Et à ce chapitre, il est certain que la diététique s'avère en meilleure posture que les sciences domestiques. Cela explique peut-être le diagnostic très sévère que pose le mémoire des diététistes sur l'enseignement ménager universitaire. On y demande notamment que le diplôme «faussement universitaire» des instituts familiaux soit aboli pour mettre un terme à la «dévalorisation» du baccalauréat et que les diplômées des instituts ne puissent enseigner les arts ménagers qu'au niveau primaire, réservant le secondaire aux détentrices d'un baccalauréat en sciences domestiques ${ }^{76}$.

À la suite du rapport Parent qui conclue, sans équivoque, à l'intérêt d'une formation professionnelle pour les filles au lieu d'une formation axée sur la famille, les diététistes semblent multiplier les gestes pour exclure de leur champ les diplômées en sciences ménagères. En 1965, le message est clair: la corporation refuse de reconnaître un cours créé à l'Hôtel-Dieu pour les diplômées des instituts familiaux afin d'en faire des techniciennes en diététique, ultime geste pour sauver ce qui reste de l'enseignement ménager et lui trouver des débouchés ${ }^{77}$. L'historienne Nicole Thivierge rapporte ainsi que:

L'Association des diététistes proteste contre cet envahissement de son domaine. La présidente de l'Association se plaint auprès du sous-ministre de la santé de «la fausse impression de compétence» que procure un cours placé sous la responsabilité de l'Association des techniciennes en sciences familiales. Elle s'interroge sur la capacité de ces techniciennes, formées à la «vie familiale», de juger de la qualité du service alimentaire d'un hôpital ${ }^{78}$.

Les diététistes nient ainsi aux spécialistes de l'enseignement ménager toute compétence pour former des techniciennes en diététique, chose qu'elles considèrent de leur ressort exclusif ${ }^{9}$. De plus,

76. Ibid., 49.

77. La Corporation en profitera pour demander à ses membres de refuser d'organiser tout programme pour techniciennes avant qu'il ne soit approuvé par elle. Bulletin $C D Q, 6,2$ (mars 1965): 9.

78. N. Thivierge, Écoles ménagères et instituts familiaux: un modèle féminin traditionnel (Québec, Institut québécois de recherche sur la culture, 1982), 385.

79. Sans y voir de contradiction, les diététistes jugent par ailleurs pouvoir présider à la formation des enseignantes d'arts ménagers au secondaire. Voir, Institut de diététique et de nutrition de l'Université de Montréal, License d'enseignement secondaire (alimentation). Projet soumis au Service de la formation des maîtres du ministère de l'Éducation (octobre 1965). 
lors du transfert de l'école domestique Saint-Pascal à l'Université Laval, on demande en 1965 que le «terme domestique soit exclu de l'appellation à cause de son sens péjoratif et que le nom actuel de l'École de sciences domestiques soit changé pour celui d'École ou de département de diététique ${ }^{80}{ }$. Plus tard, en 1971, on rappelle l'intérêt du titre «diététiste» plutôt que «diététicienne»:

Le mot diététicienne a une consonnance semblable à celle de «technicienne», appellation qui était et est encore utilisée par les diplômées soit en enseignement ménager, soit en sciences familiales; la confusion entre les deux termes aurait été plus facile pour des personnes non familières avec la formation académique et le champ d'action de ces deux groupes ${ }^{81}$.

Il s'agit donc bien d'un problème de frontières interprofessionnelles. Mais avec l'apparition des techniciennes en diététique, l'affaire semble réglée. Les diététistes affirment alors que seules les techniciennes formées au cégep sont aptes à les seconder et ajoutent:

Ceci nous amène à nous demander ce qu'il adviendra du [sic] technicien en alimentation? Dans l'esprit des diététistes présentes à notre débat sur la place du technicien en diététique dans un organigramme, ce technicien en alimentation «s'estompera» ou se «recyclera» graduellement puisqu'il n'existe plus d'Instituts familiaux. On le remplacera graduellement par le technicien en diététique auquel on confiera des responsabilités plus grandes $[\ldots]^{82}$

Les diététistes se démarquent donc progressivement des spécialistes de l'enseignement ménager et finissent par les évincer ou les exclure. Elles invoquent à la fois la spécialisation de leur champ (l'alimentation) et leur lieu de pratique (le marché du travail ou plus pécisément l'hôpital) afin de se distancer des sciences ménagères, caractérisées par leur perspective humaniste et leur emphase sur le noyau familial. Si des diplômées en sciences familiales travaillent encore quelque temps à titre d'auxiliaires des diététistes ${ }^{83}$, l'arrivée

L'Institut prend aussi la précaution de préciser qu'il a éliminé de son baccalauréat en nutrition les cours d'arts ménagers depuis 1954.

80. $\mathrm{CDQ}$, Mémoire soumis par la CDQ (chapitre de Québec) aux autorités de l'Université Laval (mars 1965), 13.

81. CPDQ, Bulletin (juin 1971).

82. Bulletin de la $C D Q, 2,4$ (décembre 1971): 6.

83. Cela semble être le cas d'une minorité d'entre elles: de $2 \%$ à $5 \%$ des diplômées des instituts familiaux deviennent aides-diététiciennes en milieu hospitalier entre 1955 et 1962 . Voir Mémoire de l'Association des instituts familiaux de la Province de Québec à la Commission royale d'enquête sur l'enseignement (1962), 48. Les appellations sont très variables: «aidesdiététiciennes», «techniciennes en alimentation», «techniciennes en sciences familiales», «techniciennes en nutrition». 
des techniciennes en nutrition permettra aux diététistes de saluer le départ définitif des spécialistes de l'enseignement ménager de leur champ de pratique. La seule porte de sortie laissée à ces dernières sera l'enseignement des arts domestiques au primaire et au secondaire; mais pour cela, il leur faudra accepter la disparition de leurs institutions spécialisées et la supervision de leur formation par les diététistes notamment ${ }^{84}$.

\section{B - Diététistes et infirmières}

Les infirmières constituent un autre groupe qui posera aux diététistes des problèmes de frontières interprofessionnelles. Dans ce cas, les tensions se présenteront davantage comme des tensions que comme des conflits ouverts.

De manière générale, les diététistes se plaignent, entre 1950 et 1980, d'être mal acceptées par les infirmières dont la place au sein de l'équipe médicale est plus ancienne et mieux assurée. Elles déplorent d'autant plus cette mésentente que les infirmières bénéficient d'un rapport privilégié avec les patients, rapport qui peut s'avérer très précieux lors d'une consultation diététique. Les tensions entre les deux groupes de professionnelles touchent aussi des points plus précis. Les diététistes, à titre de spécialistes de l'alimentation, entendent par exemple donner les cours de nutrition aux étudiantes-infirmières. Il existe d'ailleurs une tradition en ce sens, puisque plusieurs d'entre elles enseignent dans les écoles d'infirmières des hôpitaux montréalais dès $1914^{85}$. Tout au long de la période 1940-1980, elles devront cependant défendre ce qu'elles considèrent comme leur prérogative. En 1968, la Corporation des diététistes croit ainsi avoir obtenu l'appui de l'Association des infirmières de la province de Québec pour que seules ses membres donnent les cours de nutrition dans les cégeps; un an après, elle s'aperçoit que l'accord est beaucoup plus théorique qu'appliqué ${ }^{86}$.

Le ton monte un peu plus par la suite. Les diététistes dénoncent cette fois un empiètement plus flagrant sur leur champ de pratique. En 1974, l'Association des infirmières et des infirmiers de la province de

84. Il faut cependant préciser que les diététistes ne sont pas seules responsables de cette disparition puisque l'enseignement ménager est déjà moribond dans le Québec des années 1960. Malgré les tentatives désespérées de ses défenseurs pour l'adapter, cette formation traditionnelle allait disparaître devant l'urgence de préparer les filles au marché du travail. N. Thivierge, op. cit.

85. M. Lang, E. Upton, The Dietetic Profession in Canada (Canadian Dietetic Association, 1973). Il s'agit du Royal Victoria Hospital en 1914, et du Montreal General Hospital en 1919.

86. CDQ, Annual Report (1968-1969) et (1969-1970). 
Québec réclame le pouvoir de modifier les diètes thérapeutiques en l'absence du médecin et de la diététiste. Quelques mois plus tard, l'Ordre des infirmières se déclare contre le principe de réserver certains actes aux diététistes, ce qui n'améliore pas les relations entre les deux groupes ${ }^{87}$. La Corporation des diététistes réagit vigoureusement et envoie des lettres de protestation aux associations d'infirmières, à la corporation des médecins, au ministre, à l'Office des professions du Québec. Elle se montre d'autant plus véhémente que le Code des professions de 1973 vient confirmer la pratique exclusive des infirmières, mais n'accorde qu'un titre réservé aux diététistes.

De telles escarmouches resurgiront de temps en temps au cours des années 1970 entre diététistes et infirmières, mais sans déclaration de guerre ouverte. En fait, les tensions se manifestent surtout dans le milieu hospitalier. On mentionne ainsi que les relations entre les deux groupes sont beaucoup plus détendues dans les CLSC, DSC ou dans les écoles. Et l'éducation auprès du public, que les deux professions se sont donnée pour vocation, ne semble pas non plus susciter d'affrontements particuliers, malgré les occasions fréquentes de recoupement de leur champ de pratique. Tout se passe en fait comme si c'était surtout la pratique à caractère «médical» ou curatif qui suscitait l'essentiel des tensions entre les deux groupes.

Dans le cadre de la période où se situe notre analyse - soit les années 1950 à 1980 - l'affirmation du statut professionnel de la diététique et de la physiothérapie s'inscrit bien dans un rapport de pouvoir marqué par un objectif constant de clôture sociale. Cette dynamique se développe selon deux axes concurrents: elle se situe dans une ligne de verticalité lorsqu'il s'agit du rapport des diététistes et des physiothérapeutes avec les médecins ou les techniques auxiliaires, et dans une ligne d'horizontalité lorsqu'il s'agit du rapport avec des professions connexes comme celle d'infirmière.

Cependant, qu'elles revêtent les formes de l'exclusion, de la démarcation ou de l'appropriation d'actes spécifiques, les stratégies de clôture sociale pratiquées par les professions recourrent toutes à l'argument de la compétence basée sur la possession d'un savoir spécifique et institutionnalisé. Or, à travers les conflits interprofessionnels analysés, on se rend compte qu'il est plus facile, sur le plan légal, de baliser des champs de pratique que de délimiter de façon précise des champs de savoir.

87. CPDQ, Procès-verbaux, 27 mai 1974 et 30 septembre 1974. 
Quant aux rapports sociaux de sexe qui traversent l'univers des rapports professionnels, le phénomène de clôture sociale tend à revêtir, à mesure qu'on avance dans le $\mathrm{XX}^{\mathrm{e}}$ siècle, des formes plus diversifiées qu'au cours du siècle précédent. Ainsi, à l'exclusion pure et simple des femmes d'un champ professionnel donné - comme celui de la médecine par exemple - on verra se substituer des stratégies plus subtiles visant plutôt des objectifs de démarcation. Quant à la diététique et à la physiothérapie, elles répondront de plus en plus aux politiques de démarcation pratiquées à leur endroit par des tentatives d'appropriation d'actes professionnels spécifiques: plans de traitement, accès direct, etc...

Cependant, avec la multiplication des techniques paramédicales, on se rend compte que les différentes formes que revêt la clôture sociale dans l'univers professionnel transcendent de plus en plus le clivage entre les sexes. Les nouvelles formes de clivage qui marquent les rapports de la diététique et de la physiothérapie avec leurs techniques affiliées concernent des professions dont le membership est, sinon totalement, du moins majoritairement féminin. On pourrait dire à cet égard que, à l'image de bien d'autres formes de pouvoir, celui qui marque les rapports interprofessionnels intègre et dépasse tout à la fois les rapports sociaux de sexe.

Enfin, notre analyse nous amène à conclure que les professions demeurent avant tout des construits historiques dont le discours autant que la pratique s'inscrivent dans des rapports de pouvoir mouvants. Il s'agit là d'une réalité que le discours professionnel tend souvent à occulter. Il le fait dans la mesure où il revendique une légitimité morale (au-dessus de tout soupçon) et se présente d'emblée comme transcendant l'histoire et ses contingences. 